\title{
Acupuncture and pharmacopuncture are as effective as morphine or carprofen for postoperative analgesia in bitches undergoing ovariohysterectomy ${ }^{1}$
}

\author{
Stelio Pacca Loureiro Luna ${ }^{\text {I, Irene Di Martino }}{ }^{\text {II }}$, Silvia Elaine Rodolfo de Sá Lorena ${ }^{\text {III, }}$, Maria Luisa Buffo de Capua ${ }^{\text {IV }}$, Alfredo

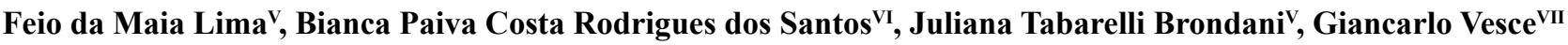

\author{
DOI: http://dx.doi.org/10.1590/S0102-865020150120000007
}

${ }^{I} \mathrm{PhD}$, Full Professor, Department of Veterinary Surgery and Anesthesiology, Faculty of Veterinary Medicine and Animal Science, Universidade Estadual Paulista (UNESP), Botucatu-SP, Brazil. Manuscript preparation.

"PhD, Department of Clinical Science, School of Veterinary Medicine, University of Naples "Federico II", Italy. Assessed postoperative pain, acquisition of data, critical revision.

IIIPhD, Unidade Acadêmica de Garanhuns, Universidade Federal Rural de Pernambuco (UFRPE), Garanhuns-PE, Brazil. Anesthetic procedures, acquisition of data, manuscript preparation.

${ }^{\mathrm{IV}} \mathrm{PhD}$, Instituto de Bioética, Botucatu-SP, Brazil. Performed the acupuncture in all animals, manuscript preparation.

${ }^{v} \mathrm{PhD}$, Department of Veterinary Surgery and Anesthesiology, Faculty of Veterinary Medicine and Animal Science, UNESP, Botucatu-SP, Brazil. Technical procedures, manuscript preparation.

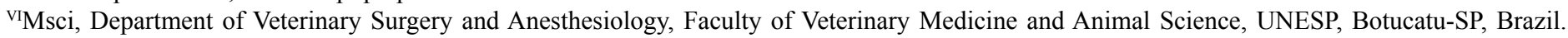
Helped in the acupuncture, anesthetic procedures, manuscript preparation.

VIIPhD, Full Professor, Department of Clinical Science, School of Veterinary Medicine, University of Naples "Federico II", Italy. Critical revision.

\section{ABSTRACT}

PURPOSE: To investigate the analgesic effect of acupuncture (AP) or micro-dose pharmacopuncture (PA), using carprofen or morphine, in bitches undergoing ovariohysterectomy (OHE).

METHODS: Thirty five dogs were randomly assigned to five groups after sedation with acepromazine IM: AP, 0.5 mg.kg-1 of morphine subcutaneously (SC), 4 mg.kg-1 of carprofen SC, and PA with 0.05 mg. $\mathrm{kg}^{-1}$ of morphine or 0.4 mg. $\mathrm{kg}^{-1}$ of carprofen. Anaesthesia was induced with propofol and maintained with isoflurane. Pain was assessed after OHE by a blind observer for $24 \mathrm{~h}$, by dynamic visual analogue scale (DIVAS), Glasgow (CMPS-SF), Melbourne (UMPS) and Colorado University pain scale (CSU). Animals reaching 33\% of the UMPS score received rescue analgesia with morphine IM. Non parametric data were analysed by Kruskal-Wallis or Friedman tests where applicable, followed by Dunn's test. Parametric data were analysed by two way ANOVA, followed by Tukey test.

RESULTS: There were no differences among groups in number of rescue analgesia. Except for the DIVAS score where animals treated with morphine had the lowest score compared with AP and carprofen, at $1 \mathrm{~h}$ after surgery, there were no other differences among groups. CONCLUSION: Acupuncture or pharmacopuncture were equally effective as morphine or carprofen to control postoperative pain in bitches undergoing ovariohysterectomy.

Key words: Hysterectomy. Pain Management. Analgesics, Opioid. Anti-Inflammatory Agents, Non-Steroidal. Dogs. 


\section{Introduction}

Non steroid anti-inflammatory drugs (NSAIDs) and opioids have been extensively used for postoperative analgesia in $\operatorname{dogs}^{1-3}$. Adverse effects of opioids may include vomiting, dosedependent respiratory depression, urinary retention, excitement/ dysphoria and histamine release ${ }^{3}$. NSAIDs should not be used in patients suffering hepatic, renal, gastrointestinal and coagulation dysfunctions ${ }^{4}$.

AP may be an alternative to avoid the adverse effects of opioids and NSAIDs in acute and chronic pain management. This technique has been successfully used in animals to treat neurological syndromes and musculoskeletal diseases which culminate in pain ${ }^{5}$ and several studies and reviews have addressed the analgesic and antinociceptive effects of AP and AP related techniques in acute pain in $\operatorname{man}^{6}$ and $\operatorname{dogs}^{7-11}$. Postoperative analgesia of AP is comparable or even better than opioids or antiinflammatory drugs ${ }^{8-11}$, showing that AP has a clinical promising effect to treat postoperative pain in dogs.

Considering that AP produces antinociception and analgesia and that in some cases NSAIDs and opioids may produce adverse effects or may be contraindicated, the replacement of analgesic drugs in pre-anaesthetic medication prior to painful procedures is a potential and attractive use of AP. This would eliminate the adverse effects of conventional analgesics and reduce treatment costs. Other beneficial effects of AP beyond analgesia, which would be relevant in the perioperative period, are reduction of postoperative nausea, vomiting and sympathoadrenal responses in man $^{6}$. Such features make AP ideal for a great range of patients, particularly those likely to develop adverse drug reactions or when adverse effects of drugs might be risky.

Although dry needle AP or electroacupunture (EA) appears to be as effective as NSAIDs and opioid analgesics for postoperative analgesia in bitches undergoing $\mathrm{OHE}^{9,11}$, maintenance of needles prior to anaesthesia might be cumbersome, particularly in agitated animals. In order to overcome this disadvantage, other techniques might be used for stimulation of acupoints. The most usually used techniques for stimulation of acupoints are application of heat (moxibustion) and low power laser AP, however both are time consuming and the last one requires specific equipment. A possible alternative would be the injection of substances or micro-clinical doses of drugs into acupoints, which is known as pharmacopuncture (PA). This method combines the traditional acupoint stimulation with the local delivery of a pharmacological agent and it is supposed to potentiate and prolong the drug effect ${ }^{12}$. Pharmacopuncture has been successfully used to sedate horses ${ }^{13}$ and $\operatorname{dogs}^{14}$.
The hypothesis of this study was that AP or micro-dose PA would be as effective as the conventional doses of NSAID or morphine to treat postoperative pain in dogs. In order to test this hypothesis this study investigated the analgesic effect of the preventive administration of AP or micro-dose PA, using carprofen or morphine, in bitches undergoing OHE. This was assessed by comparing these treatments against two positive control groups (conventional dose of carprofen or morphine).

\section{Methods}

This study was conducted under the owners' consent and after the Institutional Animal Research Ethical Committee approval. The study was prospective, randomized, and blind for pain assessment.

Thirty-five healthy bitches from several breeds were used. They aged $24 \pm 7$ months and weighed $10.1 \pm 1.7 \mathrm{~kg}$. One day before surgery all animals were evaluated and maintained in individual kennels. Selection criteria included docile, unpregnant and healthy bitches based on normal clinical and laboratorial assessment (haematocrit, total protein, alanine aminotransferase, creatinine and alkaline phosphatase). Animals were fasted for $12 \mathrm{~h}$ and water was removed for two hours prior to surgery.

All animals received acepromazine (Acepran, Univet - Vetnil, São Paulo, Brazil) 0.05 mg. $\mathrm{kg}^{-1} \mathrm{IM}$. Ten minutes later, they were randomly assigned to one of five groups. Animals from group A (acupuncture) were treated for 20 minutes with manual bilateral AP in the acupoints Gall Bladder 34 (GB34 - Yang Ling Quan), Spleen 6 (SP6 - San-yin-jiao), Stomach 36 (ST36 - Zu San Li) and Liver 3 (LIV3 - Tai Chong), using 0.25 x $30 \mathrm{~mm}$ needles (Dongban, Chungnam, Korea, Republic of South Korea). Location of the acupoints was described elsewhere ${ }^{15}$. Group M animals received $0.5 \mathrm{mg} \cdot \mathrm{kg}^{-1}$ of morphine SC (Dimorf, Cristalia, Itapira-SP, Brazil) and group $\mathrm{C}$ animals received $4 \mathrm{mg} \cdot \mathrm{kg}^{-1}$ of carprofen SC (Rimadyl, Pfizer Animal Health, Veircore Limited, Dundee, Scotland). Both were diluted in $0.9 \% \mathrm{NaCl}$ (Cloreto de Sódio, Halexlstar, Goiana-GO, Brazil) to reach a volume of 0.8 $\mathrm{mL}$. Group AM and AC were treated by bilateral PA with $0.05 \mathrm{mg} /$ $\mathrm{kg}^{-1}$ of morphine (AM) or $0.4 \mathrm{mg} / \mathrm{kg}^{-1}$ of carprofen (AC) diluted in $0.9 \% \mathrm{NaCl}$ to reach a final volume of $0.8 \mathrm{~mL}$. The total volume was subdivided in eight equal amounts of $0.1 \mathrm{~mL}$ and each amount was injected bilaterally with a 25 gauge needle at the acupoints previously described for group A.

The cephalic vein was cannulated twenty minutes after all treatments, for administration of anaesthetics and 10 $\mathrm{mL} \cdot \mathrm{kg}^{-1} \cdot \mathrm{h}^{-1}$ of Lactated Ringer (Ringer com Lactato, Segmenta, 
Glicolabor, Ribeirão Preto-SP, Brazil). Anaesthesia was induced with 4 to $6 \mathrm{mg} / \mathrm{kg}^{-1}$ of propofol IV (Propovan, Cristalia, Itapira-SP, Brazil). After endotracheal intubation, animals were connected to a circle anaesthetic system (HB Galant, HB Hospitalar, São PauloSP, Brazil), to deliver a mixture of isoflurane (Isoforine, Cristalia, Itapira-SP, Brazil) in oxygen $\left(15 \mathrm{~mL} \cdot \mathrm{kg}^{-1} \cdot \mathrm{min}^{-1}\right)$. The vaporizer setting was adjusted to maintain a surgical plane of anaesthesia judged by eye position, ocular reflexes, jaw tone, blood pressure changes, heart and respiratory rates in response to surgical stimuli. Dogs breathed spontaneously unless hypopnoea, hypercapnia or signs of inadequate anaesthetic depth occurred. Under these circumstances animals were manually ventilated.

Anaesthesia monitoring included heart rate, respiratory rate, pulse oximetry, end tidal $\mathrm{CO}_{2}$ and inspired and expired isoflurane concentration (Capnomac Ultima, Datex Engstrom, Helsinki, Finland), indirect systolic arterial blood pressure (Doppler Ultrasonic Flow Detector, Model 811-B, Parks Medical Electronics, Aloha, Oregon, USA), with the sensor placed on the metacarpal artery and rectal temperature using a standard digital thermometer. All measurements were recorded every five minutes only as control variables, therefore data are not presented in results.

After surgery the animals were moved to their kennels for recovery from anaesthesia, where the observer, blind to the treatment and experienced in pain assessment for dogs, assessed postoperative pain.

Pain measurements were performed at 1, 2, 4, 8, 12 and 24 $\mathrm{h}$ after surgery, by dynamic visual analogue scale (DIVAS), short form Glasgow composite pain scale (CMPS-SF) ${ }^{16}$, University of Melbourne pain scale (UMPS) ${ }^{17}$ and Colorado State University canine acute pain scale (CSU). DIVAS measured pain intensity by a $100 \mathrm{~mm}$ line with zero corresponding to no pain and 100 to the maximum pain.

Animals reaching 33\% of the UMPS score (nine points) received rescue analgesia with $0.5 \mathrm{mg} / \mathrm{kg}^{-1}$ of morphine IM. In these cases animals continued to be assessed and were not excluded from the statistical analysis. Seven days after surgery all bitches were clinically assessed, weighed and surgical suture was removed.

\section{Statistical analysis}

Non parametric data (UMPS, CMPS-SF, CSU scores and number of rescue analgesic treatment) were analysed by Kruskal-Wallis test followed by multiple comparisons performed by Dunn's test. Differences over time within each group were evaluated by repeated measures using Friedman test, followed by multiple comparisons using Dunn's test. Results were presented as medians and semi-ranges. Parametric data, as DIVAS score and transoperative cardiorespiratory data were analysed by repeated measure two way analysis of variance (ANOVA), with multiple comparisons and Tukey test. Animals' age and weight were analysed by one way ANOVA. Significance level was fixed at $\mathrm{p}<0.05$. Tests were performed by Sigma-Stat 3.5 software.

\section{Results}

There were no statistical differences between groups for age, body weight and propofol induction dose. Surgery and anaesthesia duration were $9 \pm 3$ and $18 \pm 6$ minutes (mean and standard deviation) respectively in all groups. Heart rate was greater in AC compared to the other groups at some time points, but values were within the normal range for the species. There were no other differences in the cardiorespiratory variables, including end tidal isoflurane concentration, during anaesthesia among groups.

Rescue analgesia was performed in 12 out of 35 bitches (34\%) (Table 1). There were no differences among groups in number of animals receiving rescue analgesia or number of times rescue analgesia was performed.

TABLE 1 - Number and time of analgesic rescues in the postoperative period of bitches undergoing ovariohysterectomy previously treated with acupuncture $(A: n=7)$; carprofen $(C: n=7)$; morphine $(M: n=7)$; pharmacopuncture with carprofen $(A C: n=7)$ or pharmacopuncture with morphine (AM: $n=7)$.

\begin{tabular}{ccccc}
\hline Group & $1 \mathrm{~h}$ & $2 \mathrm{~h}$ & $4 \mathrm{~h}$ & $\begin{array}{c}\text { Number of animals treated } \\
\text { with rescue analgesia }\end{array}$ \\
\hline A & 2 & & 2 \\
C & 3 & & 3 \\
M & & 2 & 2 \\
AC & 1 & 2 & & 3 \\
AM & 1 & & 1 & 2 \\
\hline
\end{tabular}

Pain scores by UMPS (Figure 1), CSU and CMPS-SF were not different between groups $(\mathrm{p}>0.05)$. Maximum pain scores before rescue analgesia was never above 11 in any subject (41\% of maximum score) according to UMPS and 11 (46\% of maximum score) according to CMPS-SF, but achieved $2.5(63 \%$ of maximum score) in CSU and 70 (70\% of maximum score) in DIVAS. The only difference in pain assessment between groups was at one hour after OHE, when DIVAS score was lower for 
Group $M$ when compared to $A(p<0.03)$ and $C(p<0.02)$ scores (Figure 2).

Pain scores evaluated by CMPS-SF, CSU and DIVAS were significantly greater during the early postoperative hours when compared to $24 \mathrm{~h}$ after surgery in all groups. UMPS was significantly greater at $1 \mathrm{~h}$, compared to 4 and $24 \mathrm{~h}$ after surgery in group $\mathrm{C}$ only (Figure 1).

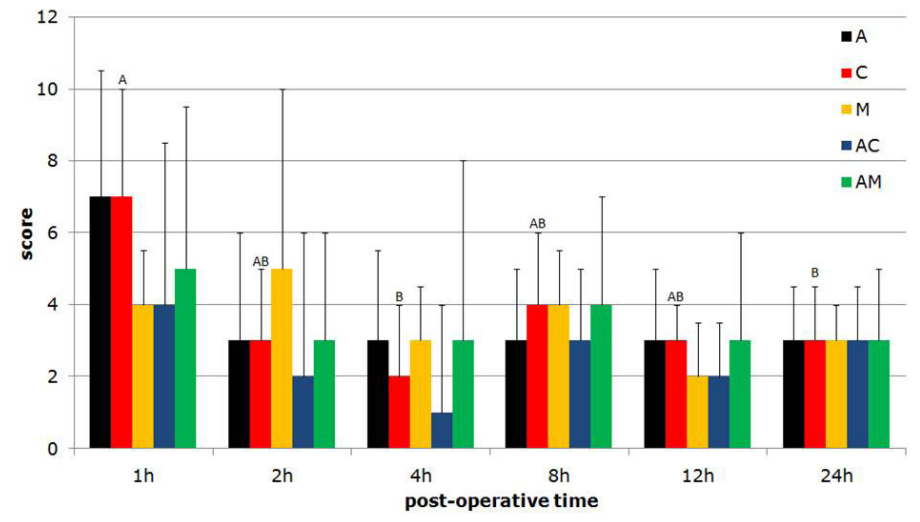

FIGURE 1 - Medians and semi-amplitudes of the University of Melbourne pain scale in bitches undergoing ovariohysterectomy. Acupuncture (A: $n=7)$; carprofen (C: $n=7)$; morphine $(M: n=7)$; pharmacopuncture with carprofen $(\mathrm{AC}: \mathrm{n}=7)$ and pharmacopuncture with morphine (AM: $n=7$ ). Capital letters expresses differences in time in the animals treated with carprofen, being $\mathrm{A}>\mathrm{B}$; maximum possible value of the scale $=27$

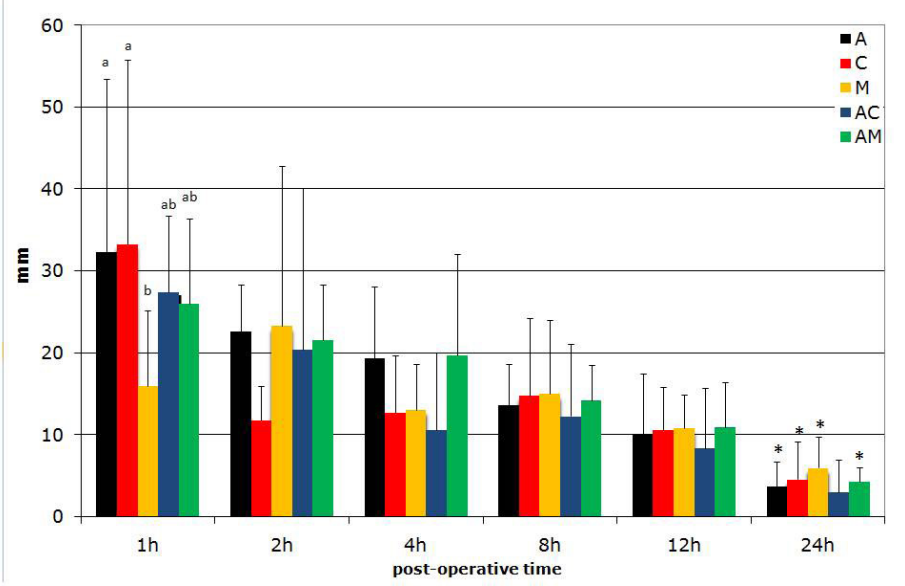

FIGURE 2 - Means and standard deviations of the dynamic and interactive visual analogue scale in bitches undergoing ovariohysterectomy. Acupuncture (A: $n=7)$; carprofen (C: $n=7)$; morphine (M: $n=7)$; pharmacopuncture with carprofen (AC: $n=7$ ) and pharmacopuncture with morphine (AM: $n=7$ ). Small letters indicate differences between groups at $1 \mathrm{~h}$ after surgery, being $\mathrm{a}>\mathrm{b}$; maximum possible value $=100$. Asterisks indicate differences between $24 \mathrm{~h}$ and other moments in each group.

\section{Discussion}

The study was designed to standardize the control variables that might interfere in the results. The surgical technique was performed by the same experienced surgeon; mean surgical time was less than 10 minutes; a minimal invasive surgical technique was performed to reduce the intensity of tissue trauma; the same anaesthetist, as well the same International Veterinary Acupuncture Society (IVAS) certified acupuncturist treated all patients; and the same experienced evaluator accomplished pain assessments.

Both AP and PA provided the same analgesic effect compared to either carprofen or morphine, confirming that AP and PA may be clinically used to minimize pain following OHE in dogs. Previous studies had already reported similar preemptive analgesic effects of conventional systemic doses of NSAIDs and opioids for postoperative pain in dogs as observed here ${ }^{1,2}$. However it is generally accepted that preventive rather than preemptive analgesia should be performed in order to avoid pain in the pre, trans and postoperative period. Differences of these two analgesic modalities have been well discussed previously ${ }^{18}$ and further research would be necessary to compare the preversus postoperative use of acupunture and pharmacopunture in controlling postoperative pain.

Pain should be assessed as a complex and multidimensional phenomenon. According to that it has been generally accepted that a multimodal treatment should be used in order to accomplish all mechanisms of pain. None of the treatments were completely effective for postoperative analgesia in bitches undergoing OHE. Rescue analgesia was necessary in all groups probably because only a single agent and dose was used preoperatively for postoperative analgesia. This might be a reflex of the unimodal analgesic treatment used in this study.

Pain response to surgery and sensitivity to analgesic therapy are unique to each individual, including genetic variation of the number, distribution and morphology of opioid receptors ${ }^{19,20}$. In general, experience of pain is highly variable across individuals, even when identical stimuli are applied under identical environmental conditions ${ }^{21}$.

Acupuncture has been successfully used to treat pain originating from a variety of disorders ${ }^{5-7,11,22}$ and a substantial part of the studies designed to explain the physiological basis of AP have been involved with pain assessment. The proposed mechanisms explaining the AP analgesic effect may be found elsewhere ${ }^{22-26}$. The selection of acupoints for this study was based on previously published studies where acupuncture successfully 
produced analgesia in $\operatorname{dogs}^{7-11}$, as well as traditional Chinese medicine indications ${ }^{15}$.

To our knowledge there are no reports investigating the injection of micro-doses of opioids or NSAIDs into acupoints. It could be postulated that the injection of a solution into an acupoint would produce a local pressure, prolonging the mechanical stimulus of AP. Pharmacopuncture might enhance the effects of the injected substances combining the traditional acupoint stimulation with the local drug delivery and drug action ${ }^{12}$. This technique has been successfully used to sedate horses, as efficiently as the conventional dose of acepromazine ${ }^{13}$. Other substances have also been used for PA in traditional Eastern medicine ${ }^{12}$.

Teleantagonism, a phenomenon based on the pharmacodynamic property of the primary nociceptive neurons, is another postulated mechanism to explain the analgesic effect of micro-dosis of carprofen and morphine injected into acupoint ${ }^{27}$. This can be explained by the fact that mechanical hypernociception elicited by intrathecal injection of NMDA, glutamate and $\mathrm{PGE}_{2}$ was inhibited by intraplantar injection of morphine and metamizole. This shows that hypernociception includes the whole neuron and there is antagonism between two substances when administered distant from one anothe ${ }^{27}$. Under these circumstances it might be postulated that the peripheral injection of both opioid and NSAID at a distal end of the nerve fiber would produce antinociception, by modification of the primary nociceptive neuron sensitivity in response to sensory stimulation. This effect is not systemically mediated, but by diffusion of the drugs along the primary nociceptive neurons, as labelled naloxone was dectected in the sciatic nerve, dorsal root ganglion and spinal cord, but not in the paw or blood after intraplantar injection ${ }^{27}$.

The peripheral analgesic effect of opioids and NSAIDs in previously inflamed tissue, when the primary nociceptive neurons are formerly sensitized, has also been described and could account for the analgesic effect of peripheral administration of microdosis of morphine and carprofen in this study ${ }^{28}$. These effects are mediated by enhancement of the nitrous oxide related pathway ${ }^{27}$.

The analgesic effect of AP covers most of the mechanisms of pain, including transduction, transmission, modulation and perception, by an anti-inflammatory, humoral and neurological effect $^{22-26}$. Acupuncture and opioids present a common mechanism, by activating inhibitory descending pathways in the spinal cord and preventing noxious stimuli to reach the brain ${ }^{24}$. Recently the American Animal Hospital Association guidelines reported that AP is a "compelling and safe method in veterinary patients and should be strongly considered as part of multimodal pain management plans" $" 19$.
Ovariohysterectomy is considered a moderate painful procedure, modifying postoperative behaviour in dogs for up to $20 \mathrm{~h}^{1}$. Except for the animals submitted to rescue analgesia, prolonged analgesia (up to $24 \mathrm{~h}$ ) was observed in most animals from all groups, showing that all protocols produced sufficient analgesia in the majority of the animals.

Four different pain scales for measuring postoperative pain associated with surgery were used in this study, to improve the quality of pain assessment. Different assessors assessing pain in different animals, at different time points might produce conflicting results ${ }^{2,16,17}$. On these grounds, a single expert assessor was chosen to simplify and uniform pain evaluation. Pain scores with UMPS, CSU and CMPS-SF did not show divergences among groups and animals, thus confirming a comparable sensitivity of each scale.

Possible limitations may be attributed to this study. The surgeon was skilled and surgery was performed in a short period, which could have mask major differences among analgesic treatments. There was no placebo group or introduction of needles in false acupoints. Placebo has not been used any more in recent studies due to obvious ethical issues and by the fact that it is well accepted that OHE is a moderate painful procedure which requires postoperative rescue analgesia, when animals do not receive postoperative pain treatment ${ }^{1}$. All treatments were preventive as it has been currently recognized that the administration of analgesics before surgery in man and dogs decreases central sensitization and postoperative analgesic requirement ${ }^{1,29}$, therefore the effect of AP and PA performed postoperatively warrants further investigation. A group of animals submitted to false AP points has also not been included because it has been reported that the analgesic effect of false AP is either absent or of low intensity when compared to the use of real points?

The UMPS was used to determine the use of rescue analgesia, based on the fact that the evaluator was more familiarized in using the scale, as this was the scale adopted in the Veterinary Hospital routine and therefore accuracy would be improved. Another possible limitation of the study was that sedation may increase the pain scores in dogs and might contribute to the use of rescue analgesia. This was probably not the case in this study, as although most of the rescue analgesia was performed at $1 \mathrm{~h}$ after the end of anaesthesia, the UMPS has been previously validated and specially developed to be used postoperatively ${ }^{17}$. According to that the possible effect of sedation in increasing the pain score is less pronounced in the UMPS, when compared to CMPS, and by this time dogs were responsive to stimulation due to the short period of anaesthesia and surgery. 
Considering that there is no information regarding the intervention point to use rescue analgesia based on the UMPS, it was decided to use $30 \%$ from the total score because this is considered the transition between light and moderate pain. This is also close to intervention score reported for the CMPS, which is $25 \%$ from the total score, i.e. $6^{16}$.

Probably the main limitation of this study was the relatively small sample size in each group. It was difficult to increase the sample size as this was a clinical study and several experimental groups were studied. The number of rescue analgesia was either two or three in all groups, showing that the analgesic effect of all protocols was similar.

Dogs receiving rescue analgesia were not removed from the data, to minimize type 2 error and to investigate duration and efficacy of rescue analgesia in treating pain. Other approach like continuing to use the same value of the pain score measured at the time point rescue analgesia was firstly administered would increase type 1 error, as well as data would not be genuine.

A strong motivation for withholding systemic analgesics in animals is the concern for their potential toxicity or adverse reactions ${ }^{3,4}$. Pharmacopunture might avoid the well-known adverse effects of standard systemic doses of opioids and NSAIDs ${ }^{3,4}$. Further clinical studies using PA and dry AP, as a promising alternative to pharmacological pain treatments, are encouraged, especially under conditions where the use of NSAIDs and/or opioids are contra-indicated.

\section{Conclusions}

Pharmacopuncture was as effective as acupuncture, and both were equally effective as either morphine or carprofen to control postoperative pain in bitches undergoing ovariohysterectomy. The clinical relevance is that PA might produce less adverse effects when compared to the use of the conventional doses of drugs. The advantage of PA when compared to dry needle AP is that the injections may be performed quickly without maintaining the needles into acupoints before surgery.

\section{References}

1. Lascelles BDX, Cripps PJ, Jones A, Waterman AE. Post-operative central hypersensitivity and pain: the pre-emptive value of pethidine for ovariohysterectomy. Pain. 1997 Dec;73(3):461-71. doi: 10.1016/ S0304-3959(97)00141-3.

2. Leece EA, Brearley JC, Harding EF. Comparison of carprofen and meloxicam for 72 hours following ovariohysterectomy in dogs. Vet Anaesth Analg. 2005 Jul;32(4):184-92. doi: 10.1111/j.14672995.2005.00207.x.
3. Pascoe PJ. Opioid analgesics. Vet Clin North Am Small Anim Pract. 2000 Jul;30(4):757-72. PMID: 10932823.

4. Luna SPL, Basílio AC, Steagall PV, Machado LP, Moutinho FQ, Takahira RK, Brandão CV. Evaluation of adverse effects of long-term oral administration of carprofen, etodolac, flunixin meglumine, ketoprofen, and meloxicam in dogs. Am J Vet Res. 2007 Mar;68(3):258-64. doi: 10.2460/ajvr.68.3.258.

5. Joaquim JG, Luna SP, Brondani JT, Torelli SR, Rahal SC, de Paula Freitas F. Comparison of decompressive surgery, electroacupuncture, and decompressive surgery followed by electroacupuncture for the treatment of dogs with intervertebral disk disease with longstanding severe neurologic deficits. J Am Vet Med Assoc. 2010 Jun 1;236(11):1225-9. doi: 10.2460/javma.236.11.1225.

6. Kotani N, Hashimoto H, Sato Y, Sessler DI, Yoshioka H, Kitayama M, Yasuda T, Matsuki A. Preoperative intradermal acupuncture reduces postoperative pain, nausea and vomiting, analgesic requirement, and sympathoadrenal responses. Anesthesiology. 2001 Aug;95(2):349-56. PMID: 11506105.

7. Cassu RN, Luna SP, Clark RM, Kronka SN. Electroacupuncture analgesia in dogs: is there a difference between uni- and bi-lateral stimulation? Vet Anaesth Analg. 2008 Jan;35(1):52-61. doi: 10.1111/j.1467-2995.2007.00347.x.

8. Cassu RN, Silva DA, Genari Filho T, Stevanin H. Electroanalgesia for the postoperative control pain in dogs. Acta Cir Bras. 2012 Jan;27(1):43-8. doi: 10.1590/S0102-86502012000100008.

9. Gakiya HH, Silva DA, Gomes J, Stevanin H, Cassu RN Electroacupuncture versus morphine for the postoperative control pain in dogs. Acta Cir Bras. 2011 Oct;26(5):346-51. doi: 10.1590/ S0102-86502011000500004.

10. Groppetti D, Pecile AM, Sacerdote P, Bronzo V, Ravasio G. Effectiveness of electroacupuncture analgesia compared with opioid administration in a dog model: a pilot study. Br J Anaesth. 2011 Oct;107(4):612-8. doi: 10.1093/bja/aer199.

11. Teixeira LR, Luna SPL, Taffarel MO, Lima AFM, Souza NR, Joaquim JG, Freitas PMC. Comparison of intrarectal ozone, ozone administered in acupoints and meloxicam for postoperative analgesia in bitches undergoing ovariohysterectomy. Vet J. 2013 Sep;197(3):794-9. doi: 10.1016/j.tvj1.2013.05.015.

12. Kim J, Kang DI. A descriptive statistical approach to the Korean pharmacopuncture therapy. J Acupunct Meridian Stud. 2010 Sept;3(3):141-9. doi: 10.1016/S2005-2901(10)60029-5.

13. Luna SP, Angeli AL, Ferreira CL, Lettry V, Scognamillo-Szabó M. Comparison of pharmacopuncture, aquapuncture and acepromazine for sedation of horses. Evid Based Complement Alternat Med. 2008 Sept;5(3):267-72. doi: 10.1093/ecam/nel096.

14. Cassu RN, Melchert A, Canoa JT, Martins PD. Sedative and clinical effects of the pharmacopuncture with xylazine in dogs. Acta Cir Bras. 2014 Jan;29(1):47-52. doi: 10.1590/S0102-86502014000100007.

15. Xie H, Preast V. Xie's Veterinary acupuncture. 1ed. Ames: Blackwell Publishing; 2007.

16. Reid J, Nolan AM, Hughes JML, Lascelles D, Pawson P, Scott EM. Development of the short-form Glasgow Composite Measure Pain Scale (CMPS-SF) and derivation of an analgesic intervention score. Anim Welf. 2007;16(S):97-104. ISSN 0962-7286.

17. Firth AM, Haldane SL. Development of a scale to evaluate postoperative pain in dogs. J Am Vet Med Assoc. 1999 Mar 1;214(5):651-9. PMID: 10088012.

18. Fantoni DT, Ida KK, Almeida TI, Ambrósio AM. A comparison of pre and post-operative vedaprofen with ketoprofen for pain control in dogs. BMC Vet Res. 2015;11:24. doi: 10.1186/s12917-015-03384.

19. Epstein M, Rodan I, Griffenhagen G, Kadrlik J, Petty M, Robertson S, Simpson W. 2015 AAHA/AAFP pain management guidelines for 
dogs and cats. J Am Anim Hosp Assoc. 2015 Mar-Apr;51(2):67-84. doi: 10.5326/JAAHA-MS-7331.

20. Landau R. One size does not fit all: genetic variability of mu-opioid receptor and postoperative morphine consumption. Anesthesiology. 2006 Aug;105(2):235-7.

21. Hansen BD. Assessment of pain in dogs: veterinary clinical studies. ILAR J. 2003;44(3):197-205. doi: 10.1093/ilar.44.3.197.

22. Cao X. Scientific bases of acupuncture analgesia. Acupunct Electrother Res. 2002;27(1):1-14. PMID: 12044016.

23. Kavoussi B, Ross BE. The neuroimmune basis of anti-inflammatory acupuncture. Integr Cancer Ther. 2007 Sept;6(3):251-7. doi: $10.1177 / 1534735407305892$.

24. Lin JG, Chen WL. Acupuncture analgesia: a review of its mechanisms of actions. Am J Chin Med. 2008;36(4):635-45. doi: 10.1142/S0192415X08006107.

25. Okada K, Kawakita K. Analgesic action of acupuncture and moxibustion: a review of unique approaches in Japan. Evid Based Complement Alternat Med. 2009 Mar;6(1):11-7. doi: 10.1093/ ecam/nem090.

26. Zijlstra FJ, van den Berg-de Lange I, Huygen FJ, Klein J. Antiinflammatory actions of acupuncture. Mediators Inflamm. 2003 Apr;12(2):59-69. doi: 10.1080/0962935031000114943.

27. Funez MI, Ferrari LF, Duarte DB, Sachs D, Cunha FQ, Lorenzetti BB, Parada CA, Ferreira SH. Teleantagonism: a pharmacodynamic property of the primary nociceptive neuron. Proc Natl Acad Sci USA. 2008 Dec 9;105(49):19038-43. doi: 10.1073/pnas.0807922105.

28. Ferreira SH, Nakamura M. II - Prostaglandin hyperalgesia: the peripheral analgesic activity of morphine, enkephalins and opioid antagonists. Prostaglandins. 1979 Aug;18(2):191-200. PMID: 230543.

29. Katz J, McCartney CJ. Current status of preemptive analgesia. Curr Opin Anaesthesiol. 2002 Aug;15(4):435-41. PMID: 17019235.

\section{Correspondence:}

Stelio Pacca Loureiro Luna

Departamento de Cirurgia Veterinária e Anestesiologia

Faculdade de Medicina Veterinária e Ciência Animal- UNESP

18618-970 Botucatu - São Paulo Brasil

Tel.: (55 14)3880-2023 / 98135-5072

Fax: (55 14)3815-6072

stelio@fmvz.unesp.br

steliopacca@gmail.com

Received: Aug 12, 2015

Review: Oct 13, 2015

Accepted: Nov 14, 2015

Conflict of interest: none

Financial source: none

${ }^{1}$ Research performed at Faculty of Veterinary Medicine and Animal Science, Universidade Estadual Paulista (UNESP), Botucatu-SP, Brazil 